\title{
Evaluating the effect of Neoadjuvant chemotherapy for esophageal Cancer using the RECIST system with shorter-axis measurements: a retrospective multicenter study
}

Yusuke Taniyama ${ }^{1 *}$, Kentaro Murakami ${ }^{2}$, Naoya Yoshida ${ }^{3}$, Kozue Takahashi ${ }^{1,4}$, Hisahiro Matsubara ${ }^{2}$, Hideo Baba ${ }^{3}$ and

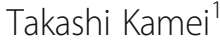

\begin{abstract}
Background: Evaluating the effect on primary lesions is important in determining treatment strategies for esophageal cancer. The Response Evaluation Criteria in Solid Tumors system, which employs the longest diameter for measuring tumors, is commonly used for evaluating treatment effects. However, the usefulness of these criteria in assessing primary esophageal tumors remains controversial. Thus, we evaluated this issue by measuring not only the longest diameter but also the shorter axis of the tumor.

Methods: We retrospectively reviewed data from 313 patients with esophageal cancer treated with neoadjuvant chemotherapy followed by esophagectomy at three major high-volume centers in Japan. All patients underwent contrast-enhanced computed tomography before and after chemotherapy. The longest and shortest tumor diameters were measured in each case. Treatment effects were adapted to the Response Evaluation Criteria in Solid Tumors system. Correlations between pathological and survival data were also analyzed.

Results: Inter-observer discrepancies were examined for changes in the longest diameter and shorter axis of the tumor (the intraclass correlation coefficients were 0.550 and 0.624 , respectively). The shorter axis was correlated with the pathological response in the multivariate analysis $(p<0.001)$. The shorter axis was significantly associated with overall survival and disease-free survival (both $p<0.001$ ), whereas this association was not observed for the longest tumor diameter.
\end{abstract}

Conclusions: This multicenter study demonstrated that the Response Evaluation Criteria in Solid Tumors system is useful for predicting pathological response and survival by incorporating the shorter axis of the primary esophageal tumor.

Keywords: Esophageal neoplasms, Neoadjuvant therapy, Response evaluation criteria in solid tumors

\footnotetext{
* Correspondence: yusuketaniyama@surg.med.tohoku.ac.jp

'Department of Surgery, Graduate School of Medicine, Tohoku University, 2-1

Seiryo-machi, Aoba-ku, Sendai, Miyagi 980-8575, Japan

Full list of author information is available at the end of the article
}

\section{$\triangle B M C$}

(c) The Author(s). 2021 Open Access This article is licensed under a Creative Commons Attribution 4.0 International License, which permits use, sharing, adaptation, distribution and reproduction in any medium or format, as long as you give appropriate credit to the original author(s) and the source, provide a link to the Creative Commons licence, and indicate if changes were made. The images or other third party material in this article are included in the article's Creative Commons licence, unless indicated otherwise in a credit line to the material. If material is not included in the article's Creative Commons licence and your intended use is not permitted by statutory regulation or exceeds the permitted use, you will need to obtain permission directly from the copyright holder. To view a copy of this licence, visit http://creativecommons.org/licenses/by/4.0/ The Creative Commons Public Domain Dedication waiver (http://creativecommons.org/publicdomain/zero/1.0/) applies to the data made available in this article, unless otherwise stated in a credit line to the data. 


\section{Background}

Neoadjuvant therapies, such as neoadjuvant chemotherapy (NAC) and chemoradiotherapy, are well-established treatments for resectable esophageal cancer treatment [1-5]. Evaluating the treatment effect before surgery is important in determining the treatment strategy because the response to neoadjuvant therapy is a well-known predictor of survival [6]. Although reports have highlighted the role of positron emission tomography (PET) and other modalities for evaluating treatment effects in esophageal cancer [7-13], these modalities are not used in clinical practice due to their cost and complexity.

In contrast, computed tomography $(\mathrm{CT})$ is commonly used in clinical settings for evaluating the response of esophageal cancer to treatment because it defines the local extent of the tumor, lymph node size, and existence of distant metastasis at relatively low cost while being reproducible and less invasive. The Response Evaluation Criteria in Solid Tumors (RECIST) (version 1.1) [14] system, which assesses the anatomical shrinkage of the tumor by measuring the longest tumor diameter, is widely used for evaluating treatment effects and can be applied to various types of tumors. However, primary esophageal tumors are occasionally classified as "nonmeasurable" because, unlike solid organ tumors, digestive organ tumors are usually not large or clear enough for appropriate measurement. Therefore, in the Japanese Classification of Esophageal Cancer (eleventh edition) [15], primary esophageal tumors have been defined as "non-measurable lesions." This is problematic in advanced primary tumors without lymph node metastases because the effect of NAC cannot be evaluated accurately. To address this, the usefulness of the RECIST system in primary esophageal tumors must be understood. Some reports [10-12] suggested that the RECIST system does not reflect actual treatment effects; however, these studies had small sample sizes and employed only the longest tumor diameter for evaluation. Few reports have evaluated this issue in a sufficiently large cohort. The purpose of this study was to clarify the usefulness of the RECIST system adapted to the primary esophageal tumor.

\section{Methods}

\section{Study design and patients}

In this retrospective multicenter study, we aimed to evaluate the utility of the RECIST system adapted to primary esophageal tumors by measuring not only the longest tumor diameter but also the shortest tumor diameter. Data from patients with esophageal cancer treated with NAC followed by subtotal esophagectomy were retrospectively collected from three high-volume centers in Japan. These centers are located in different regions and provide medical care independently. Patients with incomplete medical records, including those without precise CT scans taken before and after NAC, were excluded. Additionally, we excluded 45 patients in whom the tumor could not be identified at least $1 \mathrm{~cm}$ along the esophageal wall using CT. Finally, 313 patients were enrolled (Tohoku University Hospital [January 2009 to December 2019], $n=81$; Kumamoto University Hospital [August 2008 to April 2020], $n=149$; and Chiba University Hospital [January 2001 to December 2019], $n=83$ ). This study was approved by the appropriate ethics committees (approval numbers: 2020-1-114 [Tohoku], 2011 [Kumamoto], and 19,374 [Chiba]), and the need for written informed consent from the patients was waived.

\section{Treatment}

Patients diagnosed with stage IB/II/III esophageal squamous cell carcinoma, according to the tumor-nodemetastasis criteria, were administered NAC, as reported previously [1-5].

All patients underwent subtotal esophagectomy with radical lymphadenectomy through fifth intercostal thoracotomy or thoracoscopic procedures. The extent of lymphadenectomy was determined according to the Japan Esophageal Society (2012) guidelines [16]. Adjuvant treatment was considered for cases of non-curative resection. All specimens were pathologically diagnosed at each institutions' Pathology Department. The Union for International Cancer Control (seventh edition) [17] was used for tumor-node-metastasis staging. Briefly, stage IB corresponds to T2 without lymph node metastasis; stage II corresponds to T1 or T2 with a few lymph node regional metastases; T3 is without lymph node metastasis; stage III is associated with T1-4 with regional lymph node metastases; and stage IV is associated with distant metastasis.

\section{Measurement of tumor size on CT scan}

All patients underwent contrast-enhanced CT (arterial and venous phases) from the neck to the abdomen, with slices ranging from $0.5-5.0 \mathrm{~mm}$. CT was performed before NAC and 2-4 weeks after, and esophagectomy was performed 4-6 weeks after the last administration of NAC. The tumor area was measured at a site with different contrast enhancement and thickness compared to those of the normal esophageal wall. Tumor diameters were measured using the horizontal slice at the thickest part of the tumor. The measurements of tumor diameter before and after NAC were performed using exactly the same horizontal slice and in the same direction. The longest diameter was measured as the maximum distance from the outer tumor margin. The shorter axis was measured on the same slice as the longest diameter and was defined as the maximum tumor size orthogonal 
to the longest diameter axis; this would represent tumor thickness. In most cases, the tumor lumen could be detected using the air in the esophageal lumen, feeding tube, or normal esophageal wall; when the lumen of the tumor could not be observed clearly, we considered half of the maximal cross-sectional diameter the shorter axis.

\section{Data evaluation}

First, we examined the inter-examiner consistency of tumor size measurements by CT. We selected 81 cases (Tohoku University Hospital), and two experienced examiners (Y.T. and K.T.) measured the longest and shortest tumor diameters individually without access to the clinical data. Y.T. is a board-certified esophageal surgeon, and K.T. is a board-certified surgeon with 3 years of experience as a radiologist specializing in esophageal cancer. We then focused on the correlation between the measured treatment effect classified by the RECIST system and the clinical data, such as pathological and prognostic data. Data at each institution were measured by board-certified esophageal surgeons (Y.T., N.Y., and K.M.).

\section{Definition of treatment effect}

The RECIST system was used to classify the treatment effects using CT. Although complete response (CR) is defined as tumor disappearance, we eliminated this category because it was impossible to determine tumor disappearance without performing a biopsy during endoscopic examination. Partial response (PR) was defined as $a \geq 30 \%$ decrease in the longest diameter and shorter axis. Progressive disease (PD) was defined as an increase of $\geq 20 \%$ in these diameters. Stable disease (SD) was defined as a disease that did not meet the criteria for $P R / P D$. Since these criteria were based only on changes in the primary lesion, the changes in metastatic lymph nodes or the occurrence of new lesions after NAC were not included in the analysis.

The tumor regression grade (TRG) [18], which has been proposed by the Japan Esophageal Society, was used to evaluate the treatment effect from a pathological perspective. All specimens were classified according to a 0-5 scale based on the proportion of residual cancer cells in the primary tumor. Subsequently, this assessment was reclassified into two groups: TRG 0-1a ("ineffective group," which included patients in whom less than one-third of the tumor disappeared after treatment) and TRG 1b-3 ("effective group," which included patients in whom more than one-third of the tumor disappeared).

\section{Statistical analyses}

The intraclass correlation coefficient (ICC) was used to examine the consistency of tumor measurements between the two examiners. A receiver operating characteristic (ROC) curve was used to obtain the optimal diameter thresholds to determine the pathological response. Overall survival (OS) and disease-free survival (DFS) were analyzed using the Kaplan-Meier method and the log-rank test. OS was defined as the time from the date of surgery to the date of death from any cause. DFS was calculated from the date of surgery to the date of diagnosis of any cancer recurrence or death from any cause. Logistic regression analysis was used to assess the prediction of TRG from the treatment effect using the RECIST system. To evaluate the independent risk factors for OS according to preoperative factors and RECIST data, multivariate analysis was performed. Variables with $P<0.2$ in the univariate analysis were entered into the multivariate analysis. Statistical analyses were performed using JMP Pro 15.0.0 statistical software (SAS Institute Inc., Cary, NC, USA). $P<0.05$ was considered statistically significant.

\section{Results}

The clinical and pathological characteristics of the patients are summarized in Table 1. Among the 313 patients, $87 \%$ were male, the mean age was 66.5 years, and $89 \%$ had squamous cell carcinoma, which is similar to the proportion of patients with esophageal cancer in Japan [1]. Platinum- and taxane-based regimens were administered as NAC in 203 (65\%) and 110 (35\%) patients, respectively. In the evaluation using the RECIST system, patients were distributed equally between the PR and SD groups based on the shorter axis measurements, whereas more patients were included in the SD group based on the longest diameter measurements. TRG 01a, which represented an insufficient treatment effect on pathological examination, was observed in almost twothirds of the patients.

The examination of inter-observer discrepancies in tumor diameter measurements using CT revealed that the shorter axis was superior in evaluating changes in size (see Supplementary Table S1 in Additional File 1). The ICC value of the shorter axis was 0.624 , which is good for objectivity and reproducibility and seems to be capable of evaluating the effect of NAC. In turn, the ICC value of the longest diameter was 0.550 , which, although adequate, was not as good as that of the shorter axis.

The RECIST system showed a good correlation with the pathological treatment effect by logistic regression analysis (Table 2). In the multivariate analysis, changes in the shorter axis, rather than in the longest diameter or multiplication of both axes (tumor area), were an independent factor for TRG. This result was not affected by the tumor size (see Supplementary Table S2 in Additional File 1). Moreover, the shorter axis was associated with the pathological effect according to ROC curve 
Table 1 Patient characteristics and clinical outcome $(n=313)$

\section{Clinical, epidemiological, pathological, and surgical features}

Mean age $\pm S D$, years

$66.5 \pm 7.3$

Male sex, n (\%)

Performance status, n (\%)

0

$222(71)$

88 (28)

2

Tumor location, n (\%)

Ce

Ut

Mt

Lt

Ae

Histopathological type, n (\%)

Squamous cell carcinoma

Adenocarcinoma

$20(6)$

Others

Pretreatment clinical stage, n (\%)

II

53 (17)

$218(70)$

35 (11)

IV

Platinum-based

Taxane-based

RECIST (shorter axis), n (\%)

Severe morbidity, n (\%)

$$
c D C \geq \| l b
$$

Pathological stage, n (\%)

0-1

60 (19)

II

III

158 (41)

37 (12)

TRG

0 
Table 1 Patient characteristics and clinical outcome $(n=313)$ (Continued)

\begin{tabular}{l} 
Clinical, epidemiological, pathological, and surgical features \\
\hline 3 \\
\hline Abbreviations: $A e$ abdominal esophagus, CDC Clavien-Dindo classification, Ce cervical esophagus, Lt lower thoracic, Mt mid-thoracic, NAC neoadjuvant \\
chemotherapy, PD progressive disease, $P R$ partial response, RECIST Response Evaluation Criteria in Solid Tumors, SD stable disease, SD standard deviation, TRG \\
tumor regression grade, Ut upper thoracic
\end{tabular}

analysis (area under the curve: 0.747) (see Supplementary Table S3 in Additional File 1). The optimal shorter axis reduction rate cut-off of $32.6 \%$ had a sensitivity of $67.2 \%$, specificity of $71.0 \%$, and accuracy of $64.5 \%$. Additionally, three-dimensional measurement using the product of the shorter axis, longest diameter, and longitudinal distance showed results similar to those of the shorter axis.

Survival analyses are shown in Fig. 1 and Table 3. The mean follow-up for all patients and patients alive at the time of analysis were 1307 and 1661 days, respectively. Figure 1 shows the OS and DFS of the RECIST system classified by the longest diameter and shorter axis. The shorter axis was significantly associated with both OS and DFS, whereas the longest diameter was not. The results of univariate and multivariate analyses of OS are shown in Table 3. RECIST classification using the shorter axis was an independent prognostic factor for OS along with other factors such as TRG and pathological stage. Multiplication of the longest diameter and the shorter axis, which represents the tumor area, also correlated with OS in the univariate analysis. However, we did not include this parameter in the multivariate analysis because of its confounding effect on diameter and axis.

\section{Discussion}

This retrospective multicenter study showed that the RECIST system assessed using CT was useful for evaluating the effect of NAC on primary esophageal tumors. To allow widespread application, evaluations should be performed based on an imaging modality used in routine examinations. The advantage of using CT to evaluate the treatment effect is that this examination is necessary both before and after NAC. To determine the treatment strategy, clinicians use CT to assess tumor invasion and metastasis before starting treatment. At this point, PET is useful for detecting distant metastasis to avoid unnecessary invasive treatment. After NAC, CT is necessary for the surgeon to determine how the tumor has changed and plan surgery accordingly. However, PET is not necessary unless a new suspicious metastatic lesion is evident on CT imaging. Thus, CT is the desirable modality for evaluating the treatment effect of NAC on esophageal cancer.

Regardless of the imaging modality used, the evaluation method must also be simple enough to allow practical use (e.g., measuring the anatomical change in primary tumor size during routine examination). Although several methodologies that use CT have been studied to evaluate the treatment effect, they are too complicated to be adapted for clinical use [19-21]. The RECIST system is well known for evaluating the treatment effect, and many clinicians are familiar with it. Several reports [10-12] have used the longest diameter to evaluate the tumor by $\mathrm{CT}$, the same as in the original RECIST system; however, these have not shown good results. Moreover, since the horizontal plane of the esophagus is originally an ellipse, it is often difficult to evaluate real changes in tumor size using the longest diameter (Fig. 2). For bulky tumors, the shorter axis appears to be the most reliable measure of changes in tumor size, although the longest diameter may also be adequate. However, in cases where the tumor is not so large and is located on one side of the esophagus, the longest diameter is inadequate. Thus, the shorter axis

Table 2 Logistic regression analysis for estimating pathologically ineffective response (TRG 0-1a)

\begin{tabular}{|c|c|c|c|c|}
\hline \multirow[t]{2}{*}{ Characteristic } & \multicolumn{2}{|l|}{ Univariate analysis } & \multicolumn{2}{|l|}{ Multivariate analysis } \\
\hline & Odds ratio $(95 \% \mathrm{Cl})$ & $P$-value & Odds ratio $(95 \% \mathrm{Cl})$ & P-value \\
\hline RECIST classification by longest diameter & & $<0.001$ & & 0.888 \\
\hline$P R(v s . S D+P D)$ & $0.36(0.23-0.59)$ & & $0.95(0.43-2.32)$ & \\
\hline RECIST classification by shorter axis & & $<0.001$ & & 0.022 \\
\hline$P R(v s . S D+P D)$ & $0.24(0.15-0.39)$ & & $0.43(0.20-0.88)$ & \\
\hline${ }^{a}$ Multiplication of longest diameter by shorter axis & & $<0.001$ & & 0.112 \\
\hline$P R(v s . S D+P D)$ & $0.25(0.15-0.40)$ & & $0.45(0.17-1.21)$ & \\
\hline
\end{tabular}


multiplication of $30 \%$ reduction rate in both axes

Abbreviations: $C l$ confidence interval, $P D$ progressive disease, $P R$ partial response, RECIST Response Evaluation Criteria in Solid Tumors, SD stable disease, TRG tumor regression grade 

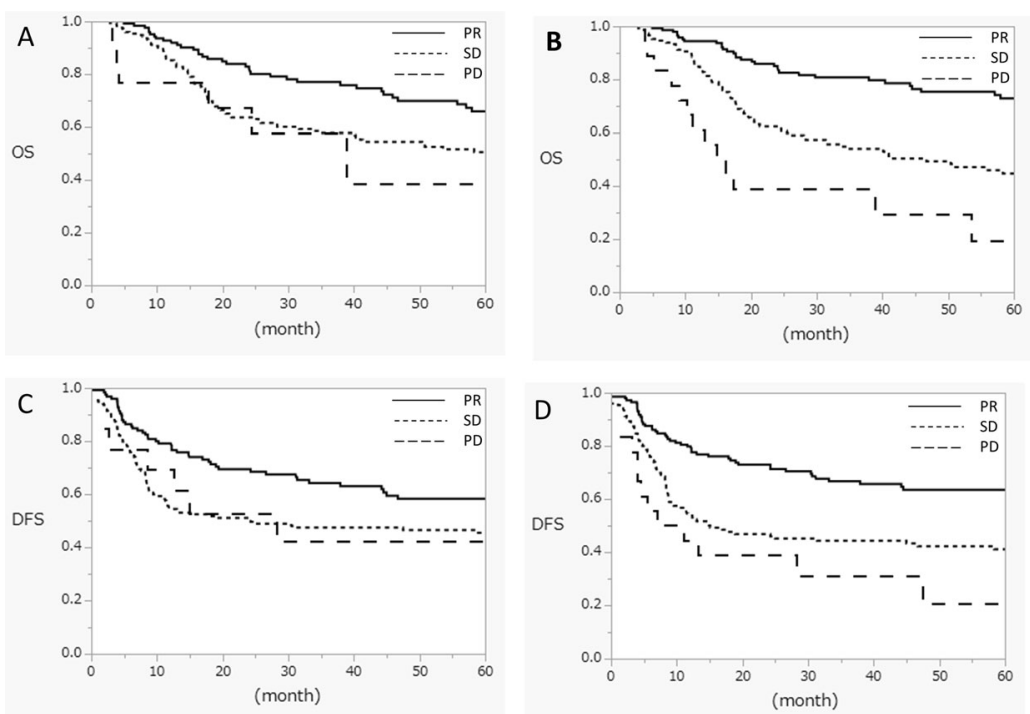

Fig. 1 Kaplan-Meier curves after esophagectomy based on the effect of neoadjuvant chemotherapy. A, B Overall survival. C, D Disease-free survival. Patients were stratified based on the RECIST system, according to changes in the longest tumor diameter $(\mathbf{A}, \mathbf{C})$ and the shortest tumor diameter (B, D. RECIST, Response Evaluation Criteria in Solid Tumors; PR, partial response; SD, stable disease; PD, progressive disease; OS, overall survival; DFS, disease-free survival. Survival analyses are described in Table 3

should be used to ease the evaluation of actual changes in tumor size.

A previous report [22] suggested that the anatomical tumor changes observed on CT could be the last phenomenon that occurs after NAC for esophageal cancer, compared to metabolic changes that are evident on PET. However, our results revealed that changes in tumor size are useful for determining the pathological response to NAC. Moreover, a 30\% reduction rate in RECIST, defined as the borderline of PR and SD, seems appropriate when the shorter axis is adapted for tumor size measurement. Our ROC curve for pathological response showed that the most appropriate cut-off value was $32.6 \%$, which was comparable to that in the RECIST system (Supplementary Table S3 in Additional File 1). These data also justify the use of the RECIST system with the shorter axis for primary esophageal tumors.

We also showed that changes in the shorter axis correlated with survival. The OS and DFS survival curves demonstrated superior outcomes in patients with PR according to the RECIST system using the shorter axis. Although the longest diameter did not demonstrate statistical significance, some trends in survival data were observed. This means that patients with apparent tumor shrinkage after NAC would have better survival, similar to the idea of pathological response. Based on these data, the measurement does not need to be performed strictly using the shorter axis; the shorter axis can be measured where the diameter appears to be the shortest. In addition, tumor area changes constitute another method for measuring the treatment effect. We evaluated this parameter by multiplying the longest diameter by the shorter axis and analyzed its correlation with the pathological effect and prognosis. Surprisingly, this multiplication parameter was inferior to the shorter axis in terms of measuring the treatment effect.

In this study, we did not use the CR criteria, an important phenomenon to be evaluated before surgery [8]. Although CT is advantageous for assessing anatomical changes and the relationships with surrounding organs, it is still impossible to detect a small number of cancer cells simply by monitoring tumor appearance. Among the 24 cases that showed tumor disappearance by CT, only four achieved pathological CR. Moreover, the four cases classified as TRG 1a did not demonstrate a sufficient response to NAC. Based on these data, pathological CR would be difficult to evaluate by CT. To evaluate the disappearance of cancer cells, biopsy during the endoscopic examination may be the best approach; however, this may still not be sufficient to determine pathological CR [23-25]. Currently, clinical CR does not reflect pathological $\mathrm{CR}$, and surgical resection is still recommended in these cases [26, 27]. Therefore, CR evaluation may not be necessary for patients receiving NAC, or "near CR" should be established as a new criterion.

The disadvantage of CT is that there is some interexaminer discrepancy compared to PET. Although our results showed that measurements of the shorter axis were relatively consistent between examiners, the ICC value was not ideal. Moreover, the issue regarding tumors that are too small to be detected by CT remains. Especially in the case of "near CR," some small tumors 


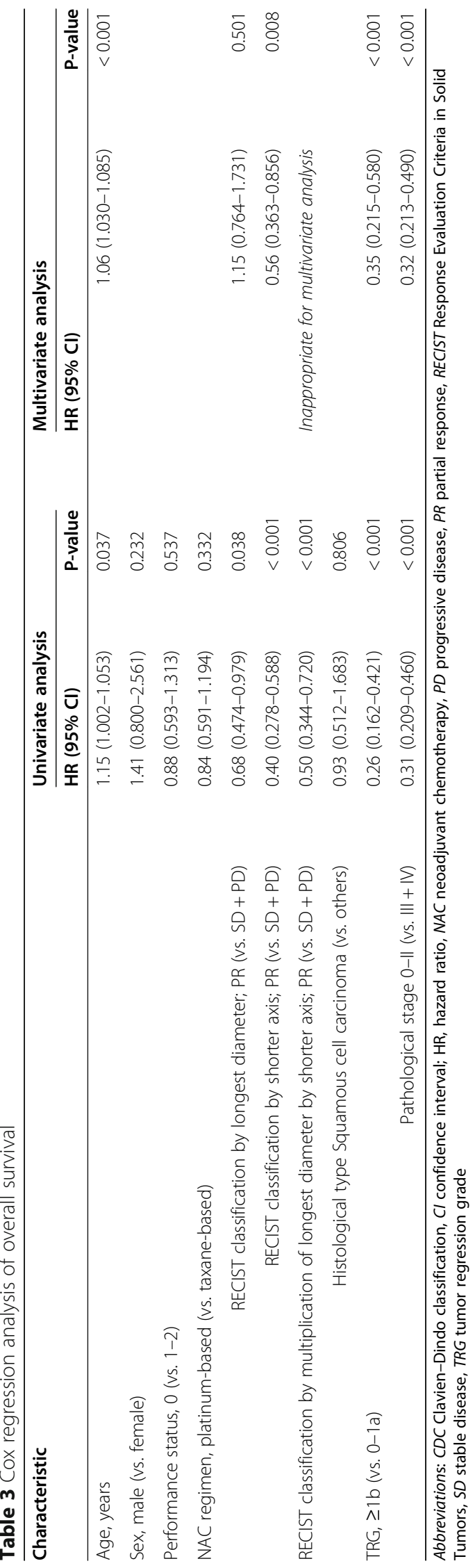




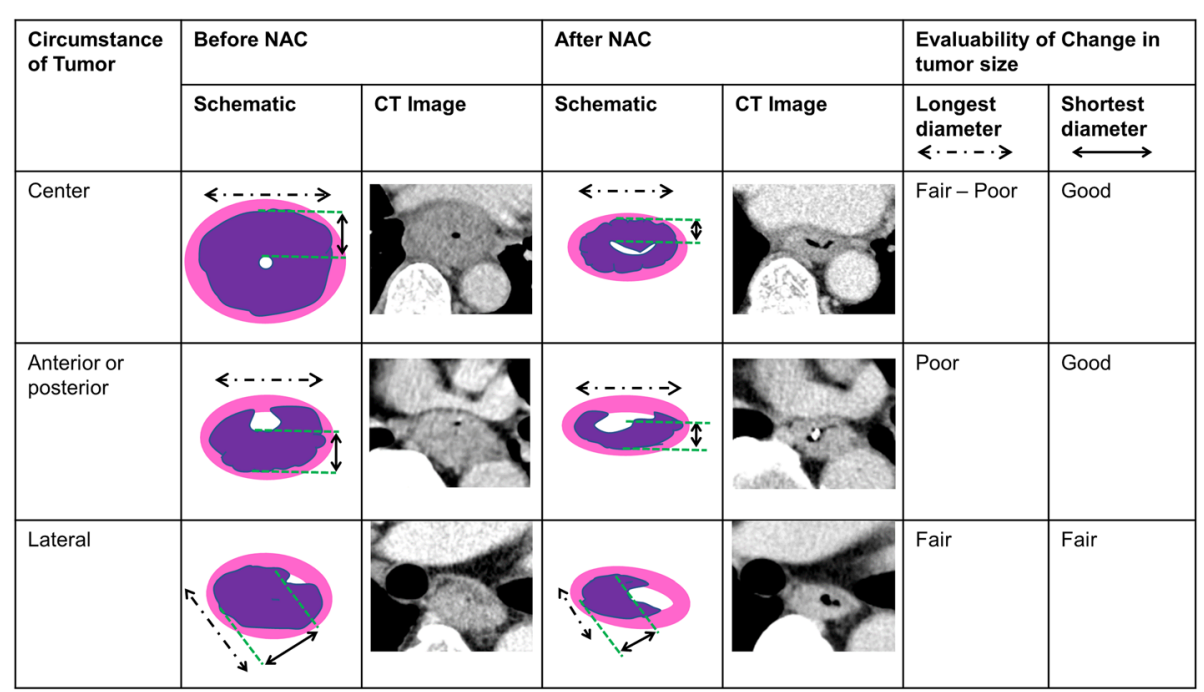

Fig. 2 Measuring changes in tumor size using the shortest and longest diameters, depending on tumor location. The longest diameter is the maximum distance between the outer tumor margins (dotted arrow). The shorter axis is the distance between the inner and outer tumor margins; it represents the tumor thickness (solid arrow). Each measurement was performed in the same horizontal slice and the same direction before and after neoadjuvant chemotherapy. NAC, neoadjuvant chemotherapy; CT, computed tomography

were difficult to identify by venous phase of CT scan, which is the standard approach in the clinical setting. Unfortunately, there are no definitive answers to these questions yet, and perhaps other modalities, such as endoscopy or PET, should be combined in such cases. However, until these modalities are commonly used before and after NAC, measurement of the shorter axis by CT remains desirable for determining treatment efficacy.

Limitations of this study include its retrospective design and the risk of inter-institutional biases in CT devices and slice width; however, we believe that these biases had little effect on our results. Moreover, other inter-institutional biases, such as differences in NAC regimens, surgical procedures, perioperative management, and treatments after recurrence, might have been present. To verify our data, validation in other studies is needed. Additionally, the accurate measurement of esophageal lesions could be quite challenging in tumors with a distorted shape. If the tumor boundary is too indistinct to be measured, it may be better to use another method; this is currently under analysis by our multicenter group.

\section{Conclusions}

This is the first multicenter study to evaluate the effect of NAC on esophageal cancer by adapting the RECIST system to assess the primary tumor. Changes in the shorter axis of the primary tumor predicted pathological response and survival. Our results support the use of the RECIST system for evaluating the effect of NAC on the primary lesion and may contribute to more consistent collection of institutional data that could be used for future research.
Abbreviations

CR: Complete response; CT: Computed tomography; DFS: Disease-free survival; ICC: Intraclass correlation coefficient; NAC: Neoadjuvant chemotherapy; OS: Overall survival; PD: Progressive disease; PET: Positron emission tomography; PR: Partial response; RECIST: Response Evaluation Criteria in Solid Tumors; ROC: Receiver operating characteristic; SD: Stable disease; TRG: Tumor regression grade

\section{Supplementary Information}

The online version contains supplementary material available at https://doi. org/10.1186/s12885-021-08747-y.

Additional file 1: Supplementary Table S1. Correlation analysis of inter-examiner variability. Supplementary Table S2. Logistic regression analysis for estimating pathologically ineffective response (TRG 0-1a) from RECIST shorter axis, divided by original tumor size (shorter axis). Supplementary Table S3. Reduction rates of the longest diameter, shorter axis, and their multiplication of the primary tumor for differentiating a pathologically "effective" (TRG $1 \mathrm{~b}-3$ ) response from an "ineffective" (TRG 0-1a) response using receiver operating characteristic curve analysis. (PPTX $46 \mathrm{~kb}$ )

Additional file 2.

\section{Acknowledgements}

The authors thank Yuichiro Doki, M.D., Ph.D., and Koji Tanaka, M.D., Ph.D. for their valuable assistance and for providing direction in performing this study. We would like to thank Editage (www.editage.com) for writing support.

\section{Authors' contributions}

All authors contributed to study conception and design. Material preparation and data collection and analysis were performed by YT, KT, KM, and NY. The first draft of the manuscript was written by $Y T$, and all authors commented on previous versions of the manuscript. All authors read and approved the final manuscript.

Funding

Not applicable. 


\section{Availability of data and materials}

The dataset supporting the conclusions of this article is included within the additional file 2 .

\section{Declarations}

\section{Ethics approval and consent to participate}

This study was approved by the appropriate ethics committees (approval numbers: 2020-1-114 [Tohoku Certified Review Board of Tohoku University], 2011 [Kumamoto University Certified Clinical Research Review Board], and 19374 [Chiba University Hospital Certified Clinical Research Review Board]), and the need for written informed consent from the patients was waived by the same ethics committee that approved the study. This study was conducted in accordance with the ethical standards of the Declaration of Helsinki of 1975.

\section{Consent for publication}

Not applicable.

\section{Competing interests}

Naoya Yoshida belongs to a department supported by Chugai Pharmaceutical Co., Ltd. and Yakuruto Honsya Co., Ltd., but has no conflict of interest to declare regarding this research. Yusuke Taniyama, Kentaro Murakami, Kozue Takahashi, Hisahiro Matsubara, Hideo Baba, and Takashi Kamei declare that they have no conflict of interest.

\section{Author details}

'Department of Surgery, Graduate School of Medicine, Tohoku University, 2-1 Seiryo-machi, Aoba-ku, Sendai, Miyagi 980-8575, Japan. ${ }^{2}$ Department of Frontier Surgery, Graduate School of Medicine, Chiba University, Chiba, Japan. ${ }^{3}$ Department of Gastroenterological Surgery, Graduate School of Medical Sciences, Kumamoto University, Kumamoto, Japan. ${ }^{4}$ Department of Radiology, Graduate School of Medicine, Tohoku University, Sendai, Japan.

Received: 25 May 2021 Accepted: 29 August 2021

Published online: 09 September 2021

\section{References}

1. Kitagawa Y, Uno T, Oyama T, Kato K, Kato H, Kawakubo H, et al. Esophageal cancer practice guidelines 2017 edited by the Japan esophageal society: part 1. Esophagus. 2019;16(1):1-24. https://doi.org/1 0.1007/s10388-018-0641-9.

2. Ando $N$, Kato $H$, Igaki $H$, Shinoda M, Ozawa S, Shimizu H, et al. A randomized trial comparing postoperative adjuvant chemotherapy with cisplatin and 5 -fluorouracil versus preoperative chemotherapy for localized advanced squamous cell carcinoma of the thoracic esophagus (JCOG9907). Ann Surg Oncol. 2012;19(1):68-74. https://doi.org/10.1245/s1 0434-011-2049-9.

3. Yokota $T$, Kato K, Hamamoto $Y$, Tsubosa $Y$, Ogawa H, Ito $Y$, et al. A 3-year overall survival update from a phase 2 study of chemoselection with DCF and subsequent conversion surgery for locally advanced unresectable esophageal cancer. Ann Surg Oncol. 2020;27(2):460-7. https://doi.org/10.124 5/s10434-019-07654-8.

4. Watanabe M, Otake R, Kozuki R, Toihata T, Takahashi K, Okamura A, et al. Recent progress in multidisciplinary treatment for patients with esophageal cancer. Surg Today. 2020;50(1):12-20. https://doi.org/10.1007/s00595-019-01 878-7.

5. Baba Y, Watanabe M, Yoshida N, Baba H. Neoadjuvant treatment for esophageal squamous cell carcinoma. World J Gastrointest Oncol. 2014;6(5): 121-8. https://doi.org/10.4251/wjgo.v6.15.121.

6. Chirieac LR, Swisher SG, Ajani JA, Komaki RR, Correa AM, Morris JS, et al. Posttherapy pathologic stage predicts survival in patients with esophageal carcinoma receiving preoperative chemoradiation. Cancer. 2005;103(7): 1347-55. https://doi.org/10.1002/cncr.20916.

7. Izumi D, Yoshida N, Watanabe M, Shiraishi S, Ishimoto T, Kosumi K, et al. Tumor/normal esophagus ratio in (18)F-fluorodeoxyglucose positron emission tomography/computed tomography for response and prognosis stratification after neoadjuvant chemotherapy for esophageal squamous cell carcinoma. J Gastroenterol. 2016;51(8):788-95. https://doi.org/10.1007/s0053 5-015-1150-4.
8. Noordman BJ, Spaander MCW, Valkema R, Wijnhoven BPL, van Berge Henegouwen MI, Shapiro J, et al. Detection of residual disease after neoadjuvant chemoradiotherapy for oesophageal cancer (preSANO): a prospective multicentre, diagnostic cohort study. Lancet Oncol. 2018;19(7): 965-74. https://doi.org/10.1016/S1470-2045(18)30201-8.

9. Tamandl D, Gore RM, Fueger B, Kinsperger P, Hejna M, Paireder M, et al. Change in volume parameters induced by neoadjuvant chemotherapy provide accurate prediction of overall survival after resection in patients with oesophageal cancer. Eur Radiol. 2016;26(2):311-21. https://doi.org/10.1 007/s00330-015-3860-7.

10. Odawara S, Kitajima K, Katsuura T, Kurahashi Y, Shinohara H, Yamakado K. Tumor response to neoadjuvant chemotherapy in patients with esophageal cancer assessed with CT and FDG-PET/CT - RECIST 1.1 vs. PERCIST 1.0. Eur J Radiol. 2018;101:65-71. https://doi.org/10.1016/j.jjrad.2018.02.009.

11. Blank S, Lordick F, Bader F, Burian M, Dobritz M, Grenacher L, et al. Posttherapeutic response evaluation by a combination of endoscopy and CT scan in esophagogastric adenocarcinoma after chemotherapy: better than its reputation. Gastric Cancer. 2015;18(2):314-25. https://doi.org/10.1007/s1 0120-014-0367-x.

12. Yanagawa M, Tatsumi M, Miyata H, Morii E, Tomiyama N, Watabe T, et al. Evaluation of response to neoadjuvant chemotherapy for esophageal cancer: PET response criteria in solid tumors versus response evaluation criteria in solid tumors. J Nucl Med. 2012;53(6):872-80. https://doi.org/10.2 967/jnumed.111.098699.

13. Lu Y, Ma L, Qin J, Wang Z, Guo J, Zhao Y, et al. The value of GRASP on DCEMRI for assessing response to neoadjuvant chemotherapy in patients with esophageal cancer. BMC Cancer. 2019;19(1):999. https://doi.org/10.1186/s12 885-019-6247-3.

14. Eisenhauer EA, Therasse P, Bogaerts J, Schwartz LH, Sargent D, Ford R, et al. New response evaluation criteria in solid tumours: revised RECIST guideline (version 1.1). Eur J Cancer. 2009;45:228-47.

15. Japan Esophageal Society. Japanese classification of esophageal cancer, 11th edition: part II and III. Esophagus. 2017;14(1):37-65. https://doi.org/10.1 007/s10388-016-0556-2.

16. Kuwano H, Nishimura Y, Oyama T, Kato H, Kitagawa Y, Kusano M, et al. Guidelines for diagnosis and treatment of carcinoma of the esophagus April 2012 edited by the Japan esophageal society. Esophagus. 2015;12(1):1-30. https://doi.org/10.1007/s10388-014-0465-1.

17. Sobin LH, Gospodarowicz MK, Wittekind C. International Union against Cancer. TNM classification of malignant tumours. 7th ed. Chichester, West Sussex, UK. Hoboken NJ: Wiley-Blackwell; 2010.

18. Japan Esophageal Society. Japanese Classification of Esophageal Cancer, 11th Edition: part I. Esophagus. 2017;14(1):1-36.

19. Lundsgaard Hansen M, Fallentin E, Lauridsen C, Law I, Federspiel B, Bæksgaard L, et al. Computed tomography (CT) perfusion as an early predictive marker for treatment response to neoadjuvant chemotherapy in gastroesophageal junction cancer and gastric cancer--a prospective study. PLoS One. 2014;9(5):e97605. https://doi.org/10.1371/journal.pone. 0097605.

20. Zhang YH, Herlin G, Rouvelas I, Nilsson M, Lundell L, Brismar TB. Texture analysis of computed tomography data using morphologic and metabolic delineation of esophageal cancer-relation to tumor type and neoadjuvant therapy response. Dis Esophagus. 2019;32:doy096.

21. Alfieri R, Pintacuda G, Cagol M, Occhipinti T, Capraro I, Scarpa M, et al. Oesophageal cancer: assessment of tumour response to chemoradiotherapy with tridimensional CT. Radiol Med. 2015;120(5):430-9. https://doi.org/10.1 007/s11547-014-0466-0.

22. Sloof GW. Response monitoring of neoadjuvant therapy using CT, EUS, and FDG-PET. Best Pract Res Clin Gastroenterol. 2006;20(5):941-57. https://doi. org/10.1016/j.bpg.2006.04.004.

23. Bohle W, Kasper M, Zoller WG. Different accuracy of endosonographic tumor staging after neoadjuvant chemotherapy and chemoradiotherapy in esophageal cancer. Surg Endosc. 2016;30(7):2922-8. https://doi.org/10.1007/ s00464-015-4578-y.

24. Fujishima F, Taniyama Y, Nakamura Y, Okamoto H, Ozawa Y, Ito K, et al. Residual carcinoma cells after chemoradiotherapy for esophageal squamous cell carcinoma patients: striving toward appropriate judgment of biopsy. Dis Esophagus. 2018;31(7). https://doi.org/10.1093/dote/dox141.

25. van Rossum PSN, Goense L, Meziani J, Reitsma JB, Siersema PD, Vleggaar FP, et al. Endoscopic biopsy and EUS for the detection of pathologic complete response after neoadjuvant chemoradiotherapy in esophageal cancer: a 
systematic review and meta-analysis. Gastrointest Endosc. 2016;83(5):866-79. https://doi.org/10.1016/j.gie.2015.11.026.

26. Piessen G, Messager M, Mirabel X, Briez N, Robb WB, Adenis A, et al. Is there a role for surgery for patients with a complete clinical response after chemoradiation for esophageal cancer? An intention-to-treat case-control study. Ann Surg. 2013;258(5):793-9. https://doi.org/10.1097/SLA. 0000000000000228.

27. Cheedella NK, Suzuki A, Xiao L, Hofstetter WL, Maru DM, Taketa T, et al. Association between clinical complete response and pathological complete response after preoperative chemoradiation in patients with gastroesophageal cancer: analysis in a large cohort. Ann Oncol. 2013;24(5): 1262-6. https://doi.org/10.1093/annonc/mds617.

\section{Publisher's Note}

Springer Nature remains neutral with regard to jurisdictional claims in published maps and institutional affiliations.

Ready to submit your research? Choose BMC and benefit from:

- fast, convenient online submission

- thorough peer review by experienced researchers in your field

- rapid publication on acceptance

- support for research data, including large and complex data types

- gold Open Access which fosters wider collaboration and increased citations

- maximum visibility for your research: over $100 \mathrm{M}$ website views per year

At $\mathrm{BMC}$, research is always in progress.

Learn more biomedcentral.com/submissions 